\title{
La educación virtual en la Universidad Central del Ecuador: ¿un nuevo reto académico?
}

\author{
Virtual Education at Universidad Central del Ecuador: \\ A New Academic Challenge?
}

A educação virtual na Universidade Central do Equador: um novo desafio acadêmico?

\author{
Javier Morillo Revelo \\ Universidad Central del Ecuador. Quito, Ecuador \\ econ6@hotmail.com \\ https://orcid.org/0000-0002-0544-2962 \\ Iván Morales Puruncaja \\ Universidad Central del Ecuador. Quito, Ecuador \\ iv.morales@hotmail.com \\ https://orcid.org/0000-0003-0193-8693
}

DOI: https://doi.org/10.32719/25506641.2022.11.6

Recibido: 15 de enero de 2021 - Revisado: 12 de marzo de 2021

Aceptado: 22 de abril de 2021 - Publicado: 1 de enero de 2022

Artículo de investigación

Licencia Creative Commons

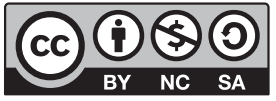




\section{Resumen}

Esta investigación trata sobre la gestión del conocimiento educativo a través del empleo de herramientas proporcionadas por las tecnologías de información y comunicación (TIC) en la Universidad Central del Ecuador (UCE), específicamente en la Facultad de Ciencias Administrativas (FCA), para analizar cómo el avance científico tecnológico incide en la educación superior en el contexto de la pandemia causada por la COVID-19. El objetivo es analizar el empleo de nuevas competencias apoyadas por las plataformas educativas virtuales, en busca de mejorar la enseñanza-aprendizaje y la capacidad autónoma de los estudiantes. El estudio es de tipo descriptivo por el enfoque teórico del tema, con respecto a la gestión del conocimiento en el aspecto educativo; por otro lado, el uso de la tipología cuantitativa se debe a la operatividad de cifras estadísticas y al empleo del método científico, con una aplicación técnica de estudio de campo a través de encuestas. La conclusión es que la gestión del conocimiento, mediante el empleo de las TIC, es el motor de apoyo en la enseñanza-aprendizaje en los actuales momentos, promoviendo el desarrollo de los docentes a través del e-learning, lo que conlleva a explotar al máximo las capacidades del alumnado en igualdad de oportunidades.

Palabras clave: TIC, virtual, e-learning, aprendizaje colaborativo, COVID-19.

JEL: O32 Gestión de la innovación tecnológica y de la I+D.

\section{Abstract}

This research addresses the management of educational knowledge using information and communication technologies (ICT) tools at Universidad Central del Ecuador (UCE), specifically at the Faculty of Administrative Sciences, to analyze how technological scientific progress influences higher education in the context of the COVID-19 pandemic. The objective is to analyze the use of new competencies backed by virtual educational platforms, in order to improve the teaching - learning process and the autonomous capacity of students. This study is descriptive in nature due to the theoretical perspective of the subject in terms of the management of knowledge in the educational field; on the other hand, the use of the quantitative typology is due to the operability of statistical figures and the use of the scientific method, with a technical application of a field study by means of surveys. The conclusion is that the use of ICTs in knowledge management is currently the support engine in the teaching-learning process, promoting the growth of teachers through e-learning, and thereby allowing them to fully exploit the capacities of students under equal opportunities.

Keywords: ICT, virtual, e-learning, collaborative learning, COVID-19.

JEL: 032 Technological Innovation Management and I+D.

\section{Resumo}

Esta pesquisa trata da gestão do conhecimento educativo através do emprego de ferramentas proporcionadas pelas tecnologias da informação e da comunicação (TIC), na 
Universidade Central do Equador (UCE), especificamente na Faculdade de Ciências Administrativas, para analisar como o avanço científico e tecnológico incide na educação superior no contexto da pandemia causada pela COVID-19. O objetivo é analisar o emprego de novas competências apoiadas pelas plataformas educativas virtuais, afim de otimizar o ensino, o aprendizado e a capacidade de autonomia dos estudantes. $\mathrm{O}$ estudo é de gênero descritivo pelo enfoque teórico do tema, no que se refere à gestão do conhecimento no âmbito educativo, por outro lado, o uso da tipologia quantitativa deve-se ao manejo de cifras estatísticas e à utilização do método científico, com uma aplicação técnica de estudo de campo através de enquetes. A conclusão é que a gestão do conhecimento, por meio da aplicação das TIC, são o motor de apoio ao ensino-aprendizado, nos momentos atuais, promovendo o desenvolvimento dos docentes, recorrendo ao e-learning, o que conduz a explorar ao máximo as capacidades dos discentes em isonomia de oportunidades.

Palavras-chave: TIC, virtual, e-learning, aprendizagem colaborativa, COVID-19.

JEL: 032 Gestão de Inovação Tecnológica e P\&D.

\section{Introducción}

$\mathrm{L}$

a educación es un proceso que evoluciona de manera constante debido a las necesidades que la sociedad presenta en el tiempo y a la par de la transformación en los avances científicos y tecnológicos; por esta razón, los docentes de la Universidad Central del Ecuador se están preparado en el campo educativo, poniendo mayor énfasis en el manejo de las plataformas virtuales.

En consonancia con lo anterior, hay que decir que para implementar un modelo educativo — donde el principal protagonista sea el docente —, tal y como lo plantean Pérez y Merino (2017, párr. 3), “deberá utilizarse el aula virtual, entendida esta como una plataforma que posibilite el desarrollo del proceso de aprendizaje creada para la interacción de los alumnos con los docentes, en la que, a su vez, haya lugar para el criterio personal”.

En esta línea, es importante tomar en cuenta que valió la pena que los maestros de todo el mundo se pregunten si el sistema educativo estaba preparado para enfrentar los problemas académicos resultantes de la pandemia por la COVID-19 y, frente a esta interrogante, es preciso mencionar que, al parecer, en el mundo entero la mayor parte de las instituciones de educación superior (IES) no lo estaban, a excepción de aquellas universidades que te- 
nían su oferta académica en la modalidad a distancia y en línea tal como lo indican Jaramillo, Casares y Vásquez (2020).

El problema de estos cambios radica principalmente en la limitada infraestructura tecnológica que pueden tener las IES, sobre todo las de carácter público en el Ecuador, debido a la enorme demanda por parte de estudiantes y profesores.

Adicionalmente a esto, se suma la falta de un modelo de planeación estratégica que permita el desarrollo del proceso de enseñanza-aprendizaje, conocer con exactitud el número de estudiantes que podrían conectarse de forma virtual, como también conocer el correcto manejo de un portafolio académico, que sirva como un complemento en el proceso de educación en línea; es así que el sector rural es uno de los sectores más afectados, por lo que no se está garantizando de manera adecuada el acceso al derecho a la educación en igualdad de condiciones de niños, niñas y adolescentes en el sector rural (Jaramillo, Casares y Vásquez 2020).

Ante el rezago de los conocimientos teóricos y prácticos sobre la utilización pedagógica de la tecnología con respecto a la tecnología misma, la generación de nuevas modalidades educativas ha motivado que diversos autores se interesen en analizar el nuevo esquema educativo para determinar sus alcances e identificar sus principales problemas.

Por otro lado, hay que puntualizar que el propósito de esta investigación es analizar el empleo de nuevas competencias apoyadas por plataformas educativas virtuales, en la búsqueda de mejorar la enseñanza del maestro y el aprendizaje y la capacidad autónoma de los estudiantes. En este sentido, es necesario destacar que la temática planteada en esta investigación es parte de la innovación tecnológica en los sistemas educativos de todos los niveles.

Así, algunos organismos internacionales promueven la educación para todos a lo largo de la vida como derecho fundamental que tiene la sociedad. La UNESCO (2013) señala que hablar de tecnologías es hablar de equipos, computadoras, dispositivos y programas. En este sentido, "es la oportunidad de reflexionar acerca de cómo estamos pensando en la educación y de qué manera los niños, jóvenes y docentes aprenden y enseñan" (Gómez, Contreras Orozco y Gutiérrez Linares 2016, 61).

La incorporación de las TIC en la educación ha logrado gestionar y apoyar las actividades de enseñanza-aprendizaje, las cuales según el Reglamento 
de Régimen Académico del Ecuador, deben estar orientadas de la siguiente manera: "1. aprendizaje en contacto con el docente, 2. aprendizaje autónomo, 3. aprendizaje práctico-experimental (que podrá ser o no en contacto con el docente)" (EC 2019, 13).

Los cambios en la educación en el nivel superior vienen dándose desde tiempo atrás, donde la tecnología ha jugado un papel muy importante dentro de los procesos de enseñanza-aprendizaje, y donde la información a través de las redes sociales, el internet, están a la orden del día. Por esta razón, la educación tradicional debe ir cambiando para fortalecer así el conocimiento virtual a nivel mundial. La siguiente afirmación hace énfasis al respecto:

Es por ello el interés de implementar proyectos encaminados a un buen manejo de habilidades, destrezas y aptitudes, tanto en docentes como estudiantes, porque actualmente la academia debe trasladar un nivel superior de enriquecimiento intelectual que sea el factor desequilibrante en solucionar los problemas que atañen a una sociedad y tan compleja como la nuestra. (Castro 2018, 1)

Por otro lado, es necesario destacar que, a partir de marzo de 2020, Ecuador y el mundo entero se ve afectado por una pandemia (la COVID-19), la misma que ha provocado cambios en lo económico, social y político, es decir, en todos los ámbitos. La Comisión Económica para América Latina (CEPAL 2020, párr. 3) indica que "en más de 190 países se han cerrado las instituciones de carácter superior con el fin de evitar la propagación del virus y mitigar su impacto".

Luego de analizar la problemática por la que atraviesan las universidades, surge la necesidad de investigar cómo los medios digitales coadyuvan dentro del proceso de enseñanza-aprendizaje y conocer cuáles han sido las mayores dificultades por las que han tenido que atravesar, tanto los docentes como los estudiantes, en esta nueva forma de generar conocimiento. Así, para el Banco Interamericano de Desarrollo (BID 2020, 2) surge la necesidad de "la creación de una plataforma tecnológica efectiva, viendo comprometidos algunos sistemas educativos y la formación de miles de estudiantes".

En este sentido vale la pena preguntarse: ¿pueden los entornos virtuales, implementados en la emergencia sanitaria por la COVID-19, superar los entornos presenciales tradicionales en la universidad? 


\section{Marco teórico}

En la presente investigación se pretende investigar sobre la transferencia del conocimiento utilizando las TIC, las que en la actualidad dan valor a la humanidad. De esta manera, la universidad se ha comprometido en mejorar los procesos de enseñanza-aprendizaje por medio de estrategias que "permitan una mejora de la calidad educativa con el propósito de que los estudiantes y profesores adquieran habilidades y conocimiento que les permita defenderse en cualquier contexto en pro de la solución de problemas" (Suasnabas 2020, 57).

La UCE cuenta con jóvenes con edades comprendidas entre los 18 y 20 años; la institución posee una plataforma Moodle con la cual se realiza la gestión de aprendizaje con todas las propuestas de implementación para la educación superior, media y básica y que responde a las necesidades creadas por la COVID-19, que obligó a migrar a una educación a distancia y en línea. Estos sistemas han obligado a plantear nuevas corrientes que se han desarrollado a través de los entornos virtuales de aprendizaje (EVA), los mismos que se han aplicado desde hace algún tiempo atrás, pero hoy por el tema de la COVID-19, se los ha diseñado para sobre llevar los problemas originados por la pandemia a finales del 2019 y todo lo que va del 2021, con procesos que abarcan desde la descripción del problema hasta alcanzar los objetivos que pretende esta investigación.

Dentro de la educación se determina a dónde se quiere llegar con el proceso de enseñanza-aprendizaje en los alumnos y docentes. Se propone con ello que los estudiantes serán divididos de acuerdo con su edad para ir aprendiendo según su capacidad. Los docentes deben estar preparados para tratar con una educación inclusiva e igualitaria y alcanzar el máximo nivel de conocimiento en cada estudiante. Los fines de la educación son establecidos tanto en la Constitución Política, la Ley Orgánica de Educación Superior (LOES) y Ley Orgánica de Educación Intercultural y su Reglamento General (EC 2020, 5).

La UCE, en calidad de institución de modalidad presencial, con el problema de salud que enfrenta el mundo entero, implementó el proceso de transición a la modalidad virtual para lo cual se invirtió en una plataforma y con esta se construyeron 7500 aulas virtuales, con la capacitación para todos los profesores en modalidad en línea (Sempértegui 2020). 
Con el crecimiento poblacional que existe en la UCE, la institución recibe, por parte del Estado, menos de USD 2500 dólares per cápita por año, tomando en cuenta que la población de jóvenes que estudian en la UCE, representan un $70 \%$, de las personas que requieren acceso a la educación superior pública (Sempértegui 2020).

$\mathrm{Al}$ analizar a la educación en los EVA, es importante conocer que "el concepto de e-learning contempla el uso de tecnologías y de internet para ofrecer una amplia gama de soluciones que faciliten el aprendizaje y mejoren el rendimiento" (FAO 2014, 5), es decir, lo que trata de diferenciar son los procesos de enseñanza-aprendizaje, lo cual básicamente se resume con el siguiente enunciado:

En este sentido, la educación virtual online, enseñanza flexible educación web, docencia en línea entre otros, es una modalidad de enseñanza-aprendizaje que consiste en el diseño, puesta en práctica y evaluación de un curso o plan formativo desarrollado a través de redes de ordenadores y puede definirse como una educación o formación ofrecida a individuos que están geográficamente dispersos o separados que interactúan en tiempos diferidos del docente empleando los recursos informáticos y de telecomunicaciones. (Area y Adell 2009, 8)

Esta nueva forma de hacer las cosas va a permitir comprobar si la sociedad en su conjunto podrá crecer en evolución tecnológica, es decir, adaptarse a "las nuevas formas de acceso a la información, teletrabajo, teleformación, telemedicina, nuevos productos y servicios basados en el conocimiento, pues son los actores que ya están instalados en nuestra sociedad, y si queremos no tropezar con ninguno de ellos, tendremos que conocerlos, y ¿por qué no?, utilizarlos" (Fundación Universitaria Iberoamericana 2006, 36).

La implementación de las TIC origina independencia en cuanto a la organización que se llega a tener como institución de carácter superior, a las decisiones que se tomen y a las acciones que se emprendan, esto ayudará a que la UCE tienda a multiplicarse y transformarse y a aprender a vivir con la tecnología, que sirva de información para los más débiles y de conocimiento para los más fuertes, así lo propone la siguiente afirmación escrita por Cedeño y Murillo $(2019,120)$ : 
Una de las características de los ambientes virtuales de aprendizaje es que cuenta con funcionalidades que permiten la comunicación fluida y activa entre los actores del proceso promoviendo nuevos roles para el docente, que se convierte en un guía y moderador, y para los estudiantes, con un papel más activo en la construcción de los conocimientos.

Para entender estos cambios en la sociedad, y sobre todo en la educación, es importante entender las tendencias y fenómenos que se han dado en el mundo, llegando a interactuar entre sí, para producir una retroalimentación a través del desarrollo tecnológico, cambios en los ciclos productivos, globalización y una nueva cultura del conocimiento.

Para finalizar esta parte, conviene decir que es importante hacer hincapié en la calidad y cantidad de los aprendizajes adquiridos más que en la estructura institucional; surge así la necesidad de utilizar la tecnología como un mecanismo que permita conocer nuevas cosas de manera diferente y en menor tiempo.

En este sentido, la UNESCO $(2008,44)$ anunció su "cooperación para promover el desarrollo educativo y comunitario mediante la tecnología de código abierto". Por su parte, para Velandia et al. $(2018,104)$ "la tecnología de código abierto se considera clave para el desarrollo social, educativo y económico y una mayor integración digital, para apoyar el desarrollo económico y social".

Esta integración digital genera un cúmulo de ideas en los docentes, los cuales pueden hacer uso de varias herramientas que se encuentran a su disposición. Se podría destacar: la web 2.0, o mejor aún, su versión más actualizada, la web 3.0, pues su uso ayuda al docente a replantear las actividades de enseñanza-aprendizaje ampliándolas y completándolas con estos nuevos recursos, los cuales a su vez le permiten al educador y al educando acceder a la información actualizada para elaborar investigaciones en bien de la sociedad.

Por lo mencionado anteriormente, es importante insertar al maestro en el desarrollo tecnológico para que este a la vez pueda integrar a los educandos en todas y cada una de las actividades planificadas dentro del proceso de enseñanza-aprendizaje, a través de la construcción del conocimiento. 


\section{¿Qué busca el e-learning?}

La educación virtual a través del e-learning pretende jugar un papel protagónico para enfrentar los nuevos desafíos de la transición a una sociedad basada en el conocimiento; dicho de otra forma, lo que se procura es actualizar los conocimientos que requiere la nueva sociedad para llevar a las poblaciones marginadas una educación con calidad, para que puedan enfrentar los retos del futuro, y la esperanza de muchos pueblos privados del acceso a los avances de la era de la globalización.

En tal virtud, el e-learning busca combinar el trabajo y el estudio, ya que para muchas personas la educación virtual ofrece varias ventajas que coadyuvan a la capacitación desde el lugar del trabajo, con lo cual se ha vinculado la educación a distancia, a través de las aulas virtuales. Estas se han constituido en poderosas herramientas que han permitido incrementar la calidad de los procesos formativos a distancia y de los estudios ofertados por este tipo de instituciones educativas, que "buscan con ello transformar la educación tradicional con el apoyo de la tecnología, pero sin perder la calidez de la interacción social, el aspecto crítico y el uso racional de contextos educativos" (Cedeño y Murillo 2019, 120).

La tecnología se utiliza dentro del diario vivir en la sociedad en correcta armonía con las acciones cotidianas del hombre contemporáneo, por lo tanto, se ha convertido, en el momento actual, en una facilitadora de acciones y valoraciones éticas, que, en unión con la comunicación, abre nuevas vías y espacios de información y diálogo entre las personas y, por ello, juntas hacen posibles las sensaciones y las vivencias.

Todo lo que proviene de las tecnologías, como el sonido, la imagen, el movimiento, el texto, etc., y que se origina en diminutos aparatos que se comunican entre sí, tiene una única finalidad: facilitar la información y la comunicación entre las personas; por esta razón, es importante analizar que las TIC crean innovadoras formas de comunicación y estas, a su vez, permiten concebir nuevas formas de aprendizaje.

Es importante resaltar que tanto estudiantes como docentes "se encuentran situados en espacios reales y virtuales de interacción entre sí, y con los demás; todos sus ámbitos laborales tienen hoy la característica de compartir 
espacios de contacto físico y de comunicación virtual" (García Muñoz, Camacho y Ancona 2012).

La incorporación de estos materiales digitales tiene como objetivo primordial superar los múltiples obstáculos y dificultades que en este campo se han presentado. Lo que se pretende con todo esto es profundizar el concepto del e-learning en la educación y diferenciar los términos con significados próximos, con el fin de utilizarlos adecuadamente en la docencia a nivel superior. Para esto lo que se busca es:

- Analizar las ventajas y desventajas como las competencias de los docentes y estudiantes en el uso del EVA, en las condiciones adecuadas para el trabajo con los aspectos pedagógicos, comunicacionales, culturales y tecnológicos.

- Evidenciar el uso de las TIC en el campo de las ciencias administrativas como herramienta que nos ayuda a evaluar los resultados del aprendizaje.

- Evaluar las experiencias en el proceso de enseñanza-aprendizaje a través de los EVA para reforzar el modelo de la educación virtual en la UCE.

En este enfoque es necesario analizar la dinámica social generada por todos los agentes, autoridades, trabajadores, docentes y estudiantes, de acuerdo con su relación intermodal con los elementos, como son el mundo no humano, la sociedad global, la sociedad local, los gobiernos y las instituciones de fomento, entre otros.

\section{Metodología}

Es una investigación descriptiva de corte cuantitativo y cualitativo. Se busca comprender la realidad abordada aplicando la fórmula de la muestra con el valor $\mathrm{z}=1,76$, y la probabilidad positiva del $86 \%$, y una probabilidad negativa del $14 \%$ (conociendo que el $86 \%$ de la población antes de la pandemia estudiaba de forma presencial, mientras que el $14 \%$ lo hacía en educación a distancia), con un error del $5 \%$. En este sentido, se realizó una encuesta en línea a 178 estudiantes de una población total de 4641 jóvenes de la FCA. 
A través del programa de SPSS se seleccionó de forma aleatoria a los 178 participantes a los cuales se les aplicó la encuesta por medio del Moodle, obteniendo datos que permiten estudiar las variables a medir como son la utilización de aulas virtuales, materiales de sitios web, si los contenidos planteados por los docentes presentan el interés oportuno, así como también las herramientas que se utilizan al momento de evaluar y obtener los resultados esperados.

Para llegar a conocer el nivel de competencia que han desarrollado los docentes en el manejo de las aulas virtuales, se elaboraron dos cuestionarios, uno al inicio del semestre y, el otro, al finalizar, en los cuales los estudiantes indican las condiciones en las que se desarrolló la actividad académica tomando en cuenta algunas consideraciones básicas, como los planes de internet a los que han tenido que acceder tanto profesores como estudiantes. La mayoría de los alumnos disponen de dispositivos móviles y muchos docentes y estudiantes disponen de un solo equipo de computación para todos los miembros de la familia. Sin lugar a dudas, esto dificulta el desarrollo de habilidades en las TIC, como también el aprendizaje en el estudiante.

Con el tamaño de la muestra de los estudiantes de la FCA, se aplicó una encuesta en la cual se plantearon algunas interrogantes de las que se indicó en párrafos anteriores, para obtener información relevante sobre la causa y efecto de la utilización de los EVA. Se llegó a indagar sobre el uso de las aulas virtuales, apoyo bibliográfico a través de esta herramienta y cuál fue el resultado del aprendizaje de la metodología utilizada por parte del tutor, como también si los conocimientos adquiridos fueron los más acertados en el semestre en estudio. Por otro lado, se utilizó la información que fue descrita por la Dirección de Desarrollo Académico (DDA) en el V Curso de Aula Virtual Centralina, dictado en agosto de 2020.

\section{Análisis y resultados de la investigación}

En las declaraciones hechas por el rector de la universidad, Fernando Sempértegui (2020), indica que existen cerca de 3500 estudiantes que no tenían conectividad, para lo cual la UCE les proporcionó de este servicio, es decir, que donde quiera que se encuentre el estudiante va a poder conectarse 
a sus clases diarias. Vale la pena indicar que existe un alto porcentaje de jóvenes que se encuentran en provincias; de esta manera, existe un pequeño grupo de estudiantes que se han quedado sin acceso a la educación.

La FCA oferta tres carreras: Administración de Empresas, Contabilidad y Auditoría y Administración Pública, por lo tanto, los recursos humanos y materiales que se utilizan son propios de la facultad. En el recurso humano se tiene a los docentes, más el personal administrativo y de apoyo, los cuales se encuentran comprometidos con las exigencias que demanda la institución, conociendo que la eficacia y la eficiencia dependerá en gran parte de la formación, capacidades y actitudes del personal.

La sistematización que arrojan los resultados es la que responde a las necesidades de brindar una exposición de carácter gráfico de las encuestas realizadas a los estudiantes de la FCA. Con esto se pretende establecer el propósito de las tareas que realiza el alumno en el entorno social, como también conocer el criterio que tienen los docentes sobre el proceso educativo de la clase. Se pretende que la interacción entre el profesor y el alumno durante el proceso de enseñanza- aprendizaje sea el más adecuado. Por lo tanto, los resultados obtenidos de acuerdo con la información recogida en la investigación planteada, se analizarán en función de los objetivos planteados.

\section{Discusión}

De los resultados obtenidos se desprenden algunas interrogantes, como el uso de las TIC, que ha ido presentando dimensiones en algunos niveles que a nuestro juicio son insuficientes. En estos niveles se puede observar el grado de competencia que tienen los docentes para el uso didáctico de la tecnología y, por otro lado, los docentes que consideran que su competencia en el desarrollo y uso de las herramientas tecnológicas es moderada.

Los resultados que se obtuvieron según la información recogida en la investigación planteada, y en función de los objetivos propuestos, determina que los estudiantes, en relación con la utilización de aulas virtuales y apoyo bibliográfico, como materiales y sitios web, mostró que los docentes han participado utilizando y desarrollando sus clases de manera en línea y con el apoyo bibliográfico y sitios web tal como se puede apreciar en la figura 1. 


\section{Figura 1}

\section{Utilización de aulas virtuales, apoyo bibliográfico y materiales de sitios web}

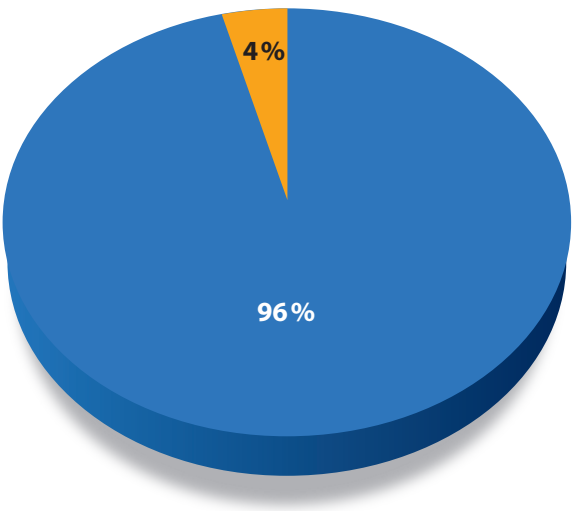

Sí

No

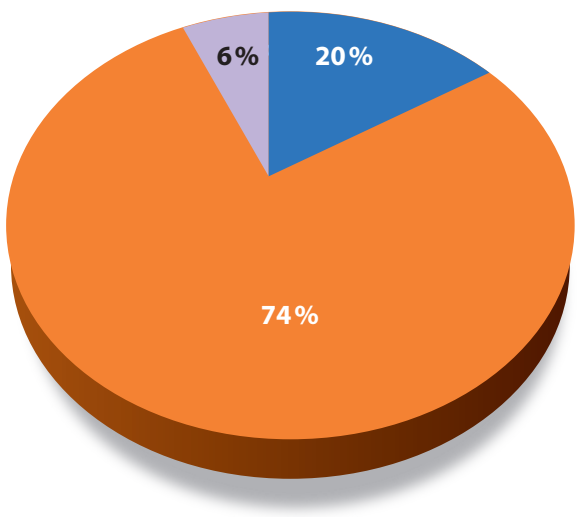

Siempre

Algunas veces

Nunca

Elaboración propia.

La figura 1 muestra que existe un $95,62 \%$ de profesores que utilizaron aulas virtuales en el semestre anterior y el 4,38\% no las usó debido a factores como la falta de internet o falta de conocimiento en el uso de las aulas virtuales. Esto significa que los recursos utilizados fueron los adecuados.

En los recursos educativos utilizados por parte del docente y en lo que tiene que ver con la bibliografía, pasa de procesos analógicos a procesos digitales, es decir, los contenidos ahora son interactivos, lo que significa que la relación de comunicación es más participativa. En este sentido, se llegó a conocer que existe un $74 \%$ que utilizó algunas veces material bibliográfico y citas a través de un sitio web, el 20\% siempre utilizó material adecuado para la realización de las clases, y apenas el $6 \%$ de docentes nunca hizo útil algún sitio o biblioteca virtual, lo que deja ver que el educando necesita mayor capacitación con respecto a este tema. 
Por otro lado, al preguntar sobre la utilización de la plataforma Moodle, si esta fue adecuada en el proceso de enseñanza-aprendizaje y si, además, los contenidos despiertan interés por aprender, contestaron lo siguiente: el $82,48 \%$ considera que es muy favorable la utilización de esta herramienta en el proceso de enseñanza-aprendizaje, ya que motiva a los alumnos la diversidad de recursos utilizados en el aula virtual, en tanto que el 5,12\% no lo considera eficaz y el $12,41 \%$ indica que a veces es bueno la utilización de este espacio, como se puede observar en la figura 2.

\section{Figura 2}

Uso de la plataforma Moodle en el proceso de enseñanza-aprendizaje y contenidos que despiertan interés por aprender

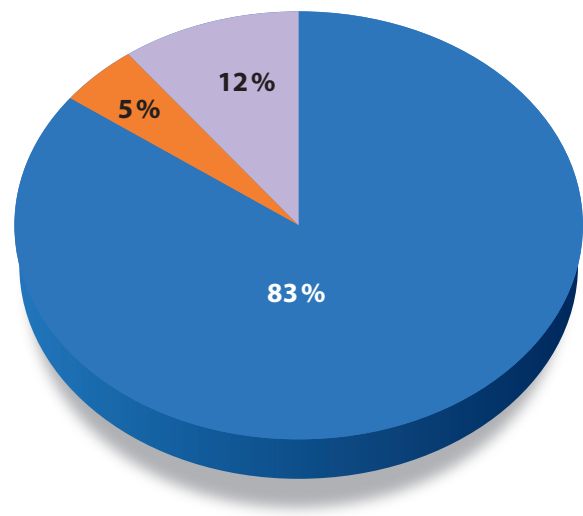

- Sí

No

$A$ veces

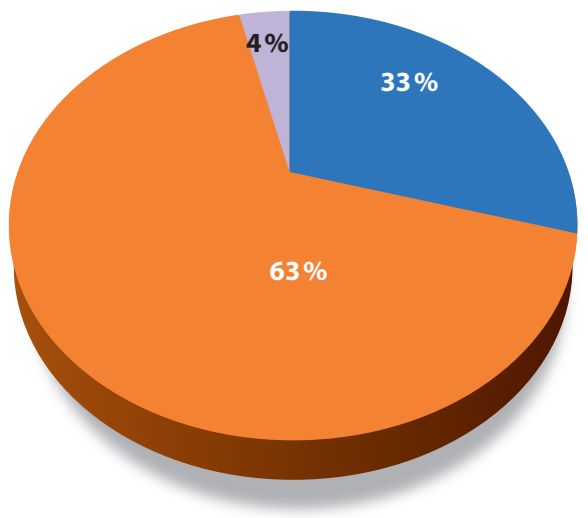

Siempre

Algunas veces

Nunca

Elaboración propia. 
Del mismo modo, al preguntar si los contenidos que exponen los profesores despiertan algún interés sobre los estudiantes, manifestaron: el 69\% de los contenidos muestran algún tipo de interés sobre los estudiantes, ya que los contenidos interactivos promueven el interés por aprender por parte del estudiante; el $26 \%$, indica que sí existe motivación con los contenidos por parte de los docentes, en tanto que el $5 \%$ no muestra ningún tipo de incentivo al estudiante, eso significa que se tiene que preparar más al docente para que conozca qué recursos son los más adecuados para el estudiante para motivar el proceso de enseñanza-aprendizaje del estudiante.

En este sentido, es importante indicar que el estudiante necesita conectarse a un dispositivo para observar su clase. De esta manera, al preguntar qué es lo que más utiliza en sus clases, como las herramientas que utiliza el docente al momento de evaluar, se obtuvo las siguientes respuestas: el $53 \%$ de los estudiantes utilizan una computadora personal, mientras que el $44 \%$ utiliza el celular. Los demás porcentajes nos indican que en la UCE apenas se utiliza el $2 \%$ las computadoras, en tanto que el $1 \%$ lo hace a través de una tablet. Esto quiere decir que los estudiantes que utilizan el celular tienen una desventaja al momento de realizar casos prácticos, debido a que dicho dispositivo le permite observar una clase, pero no le permite realizar de manera adecuada un documento que lo realizaría de forma sencilla con la utilización de un computador.

Por otro lado, al preguntar si los profesores utilizaron herramientas virtuales en el momento de evaluar se obtuvieron los siguientes resultados: el $80 \%$ algunas veces utilizaron herramientas virtuales para poder evaluar, mientras que el $10 \%$ no hicieron evaluaciones con ninguna herramienta tecnológica; eso nos indica que el docente necesita mayor conocimiento sobre el uso de las TIC, como por ejemplo Kahoot, que es una plataforma en línea que mezcla juego con entretenimiento y diversión y permite crear cuestionarios en línea en los que los estudiantes pueden participar para desarrollar actividades académicas, tal como se puede apreciar en la figura 3 .

En lo que tiene que ver con la estructura del aula virtual, se obtuvieron los siguientes resultados que se observan en la figura 4 . 
Figura 3

\section{Dispositivos que se utilizan para acceder a las clases virtuales $y$ herramientas que utilizan en el momento de evaluar}
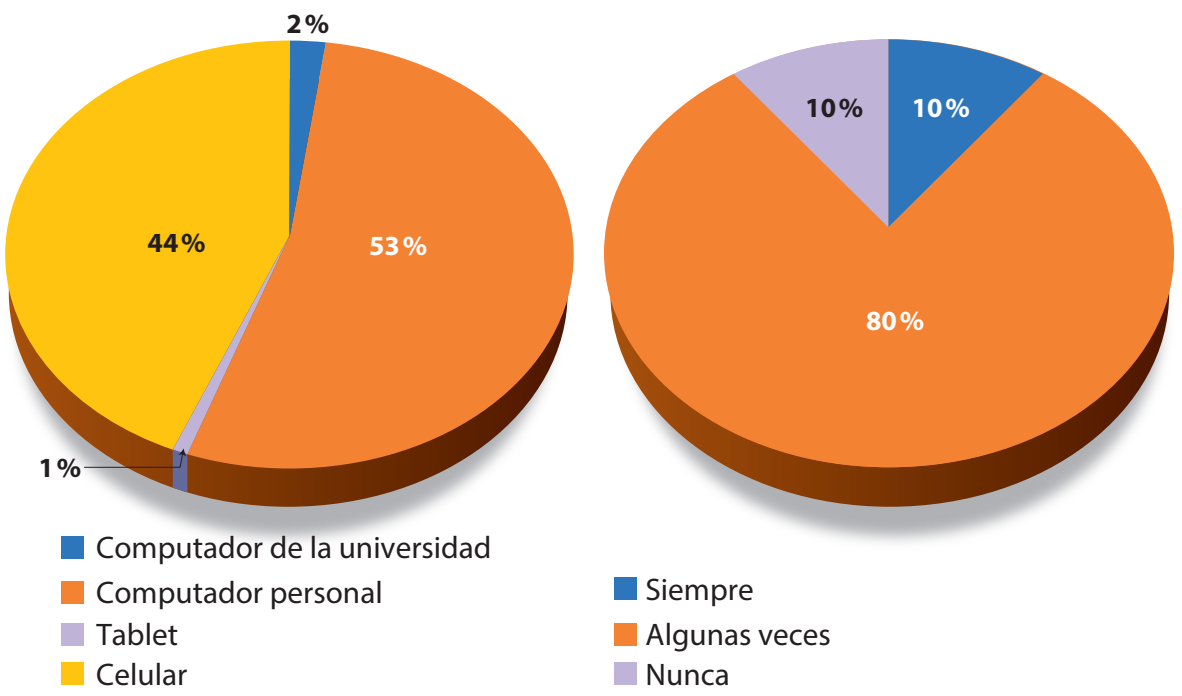

Elaboración propia.

Con la información obtenida se llegó a determinar que existe un 58,27\% que está de acuerdo con dicha estructura, mientras que el 18,11\% está totalmente de acuerdo, y apenas el 1,57\% no está de acuerdo, esto significa que los docentes pueden mejorar sus niveles de competencia con referencia a la presentación del aula virtual, dando así mejores facilidades de acceso a los estudiantes a los documentos ubicados en el aula virtual.

Por otro lado, al analizar las presentaciones realizadas por los docentes, es decir, el material, el 54,33\% está de acuerdo; significa que la mayor parte de docentes en el corto plazo tuvieron la capacidad de elaborar documentos resumidos, los que permitió una mejor comprensión por parte de los estudiantes, utilizando herramientas como Genially, tomando en cuenta que el dominio de estas tecnológicas no fue una de las fortalezas por parte del profesorado. 
Figura 4

\section{Estructura de la clase en el aula virtual y las presentaciones}
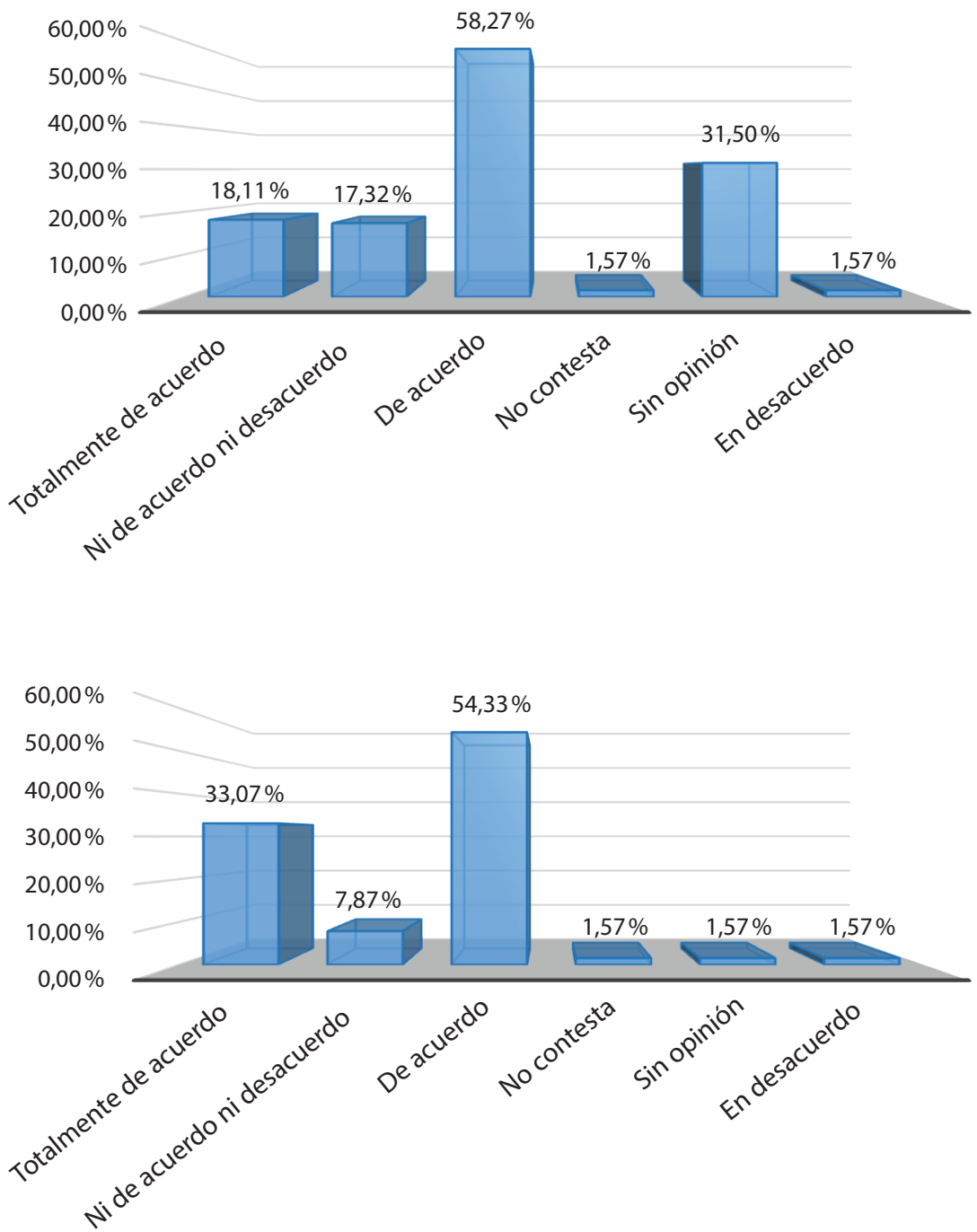

Elaboración propia. 
Figura 5

\section{Sistema de videoconferencia y materiales de apoyo}
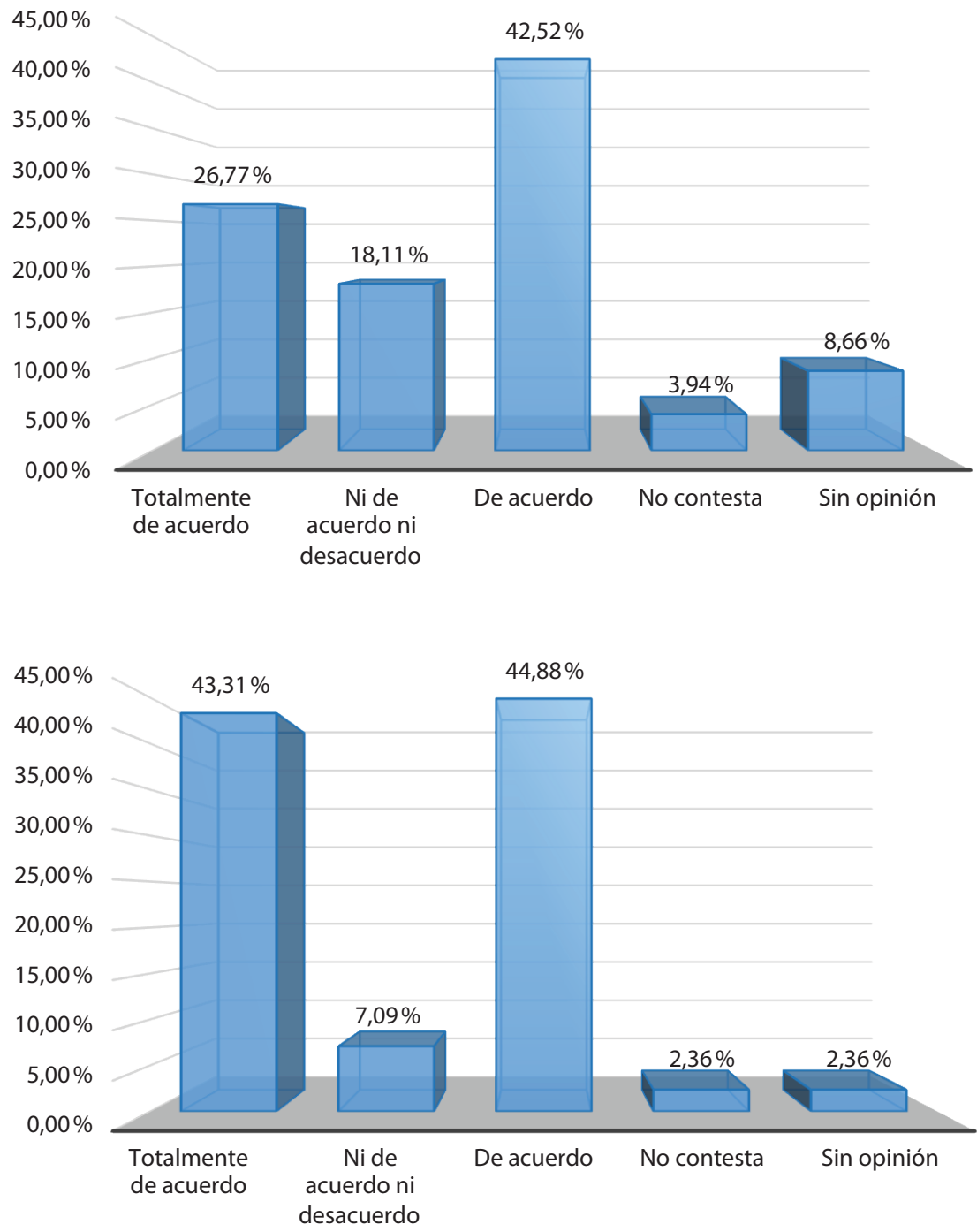

Elaboración propia. 
Del mismo modo, al referirnos al sistema de video-conferencia y materiales de apoyo a través de la herramienta Teams, se llegó a obtener la información que se observa en la figura 5.

Analizando los resultados, se obtiene que el $42,52 \%$, de las personas encuestadas están de acuerdo con el sistema de video-conferencia, esto quiere decir que la herramienta Teams es buena para elaborar las clases en forma en línea, sin dejar de lado que la utilización de otras como Zoom y Meet, son adecuadas en el proceso de enseñanza-aprendizaje.

Sobre el material de apoyo realizado por todos los docentes, el 44,88\% de los estudiantes encuestados está de acuerdo, es decir, que el nivel de competencia en los documentos realizados fueron los más adecuados, tomando en cuenta la utilización de recursos como: YouTube, Teams, OneDrive, Video Scribe, entre otros.

En lo que tiene que ver con las tareas y las evaluaciones, se determina que el $48,82 \%$ de las tareas enviadas por los docentes estuvieron de acuerdo, eso significa que desempeño y cumplimiento de estas por parte del estudiante fueron acertados, tal como se puede observar en la figura 6 .

Del mismo modo, las evaluaciones representan el $46,46 \%$, esto quiere decir que el tutor utilizó herramientas como pizarras digitales, las que permiten ir creando el conocimiento entre todos los participantes. También se utilizaron cuestionarios en línea, foros, chats, etc. Este análisis permite identificar los niveles de competencia que llegaron a obtener los docentes de la FCA. En el corto plazo se consideraron como competentes en un nivel intermedio, es decir, relacionados con las redes sociales y con las aulas virtuales.

Por lo tanto, contrastando lo dicho por Casal Otero, Fernández Morante y Cebreiro López $(2018$, 9), según los cuales "los docentes de 34 a 40 años indicaron tener un nivel de competencia mayor para poder seleccionar las TIC más adecuadas en función de los objetivos y modelos de enseñanza aplicados", los docentes que tienen mayor experiencia son aquellos que han desarrollado otro tipo de destrezas como: mejor manejo de las presentaciones en clase y presentación a los estudiantes de casos basados en las experiencias vividas en el ámbito profesional. 
Figura 6

Tareas y evaluaciones
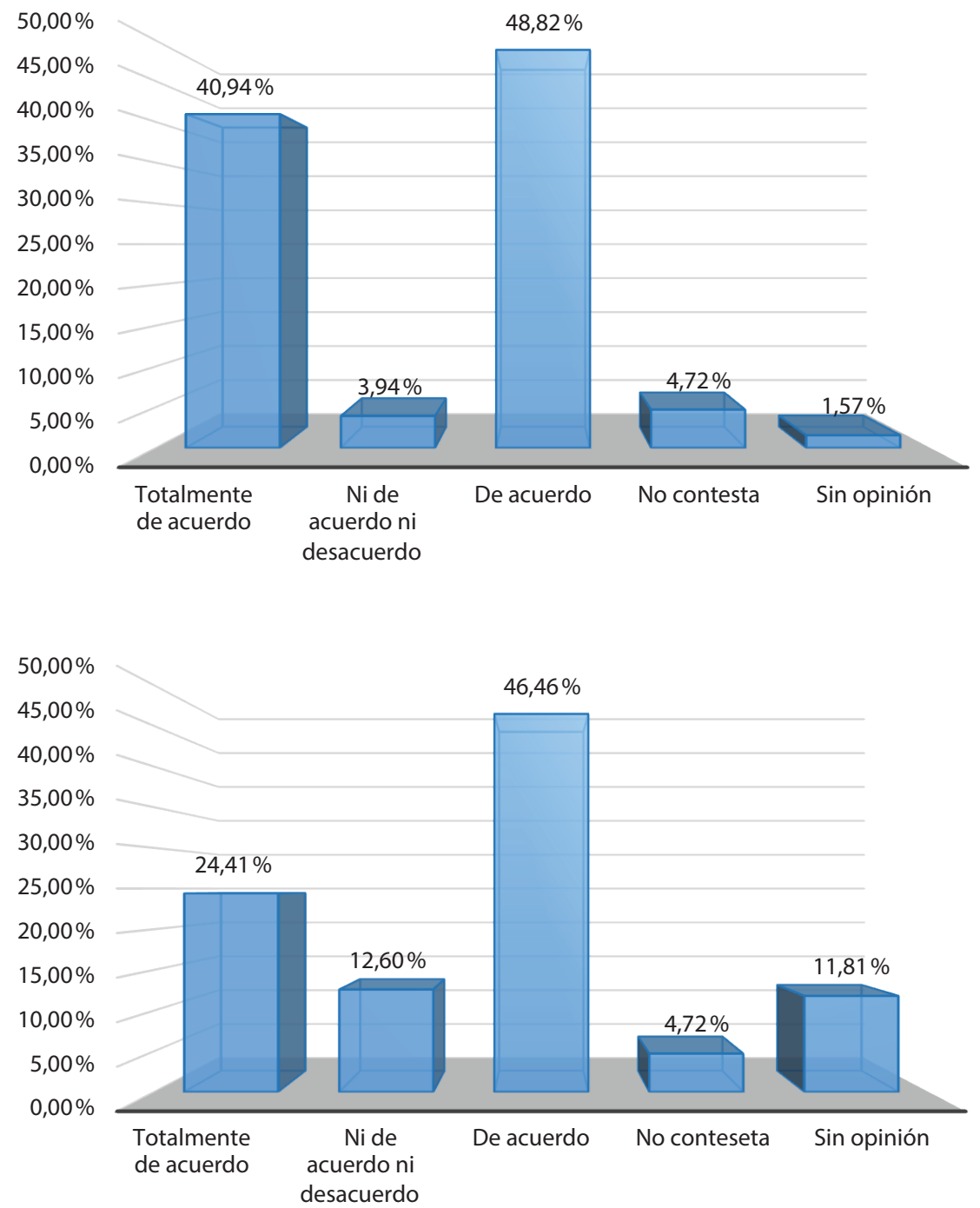

Elaboración propia. 


\section{Conclusiones}

La nueva forma de generar el proceso de enseñanza-aprendizaje a través de los EVA ha originado que los docentes se preparen para impartir conocimiento en la búsqueda de la calidad de educación, en procura de satisfacer las necesidades de los estudiantes y en el marco de una educación integral. Siendo así, en el marco del propósito principal de la investigación, que consiste en el análisis sobre el empleo de competencias digitales mediante el empleo de plataformas educativas que coadyuvan y propenden a mejorar las capacidades académicas tanto en docentes como en estudiantes, la UCE a través de la DDA viene realizado una serie de cursos para todos los docentes, al mismo tiempo que sirve para dotar de nuevas herramientas, sirve para impartir el conocimiento a través del uso de los EVA, lo que lleva a la reflexión sobre el impacto social que origina a nivel institucional y sobre el proceso de enseñanza-aprendizaje.

Como resultado, se alcanza las conclusiones basadas en la opinión de los estudiantes, que explican que la educación virtual es aquella que no posee distancias y a la que se accede por medio del uso de las TIC, de un computador y del acceso al internet.

La calidad de la educación por medio de esta nueva forma de generar conocimiento viene dada por los términos de equidad, eficiencia e innovación tecnológica, que permite la transformación permanente a través de la evaluación y el uso adecuado de herramientas y logra mejorar el proceso de enseñanza-aprendizaje. En este sentido, las conclusiones a las que llega la investigación determinan que:

En el proceso de enseñanza-aprendizaje, el constructivismo es una de las teorías en la cual el docente deja de ser el centro principal del proceso, para convertirse en el guía o en un tutor, capaz de generar en su aula un ambiente de aprendizaje, poniendo en práctica las habilidades y destrezas que todo individuo puede llegar a desarrollar con el uso adecuado de las herramientas tecnológicas, llegando así a satisfacer las necesidades que tienen los educandos y superando de esta manera las expectativas en relación con las ofertas académicas hechas por la universidad.

El uso de las TIC nos indica que la UCE se encuentra en proceso de cambio de la forma tradicional de educación a la utilización de los EVA, que 
permiten generar nuevos conocimientos en los docentes, proponiendo este nuevo modelo en el que el estudiante se autoeduque en función de generar nuevo conocimiento a través del constructivismo que en el futuro generará grandes potenciales para la sociedad.

La falta de un modelo de planeación estratégica ha impedido un mejor desarrollo del proceso de enseñanza-aprendizaje, a través del aula virtual, lo que ha dado como resultado que tanto docentes como estudiantes manejen su portafolio académico, que sirve como un complemento en el proceso de educación en línea.

Tanto la UCE como la FCA, se han visto en la necesidad de trabajar en estos nuevos escenarios de educación en línea, que permiten generar un nuevo modelo pedagógico a través del uso de las herramientas tecnológicas.

El uso de recursos tecnológicos permite al estudiante motivarse por la lectura, ofreciéndole así nuevas formas de presentación del material didáctico con formatos animados, insertar videos y usar material audiovisual, cuyo objetivo principal es apoyar los procesos de enseñanza-aprendizaje y desarrollar nuevas competencias para el estudiante.

\section{Referencias}

Area, Manuel, y Jordi Adell. 2009. E-Learning: enseñar y aprender en espacios virtuales. La tecnología educativa en el siglo XX. Archidona: Aljibe.

Banco Interamericano de Desarrollo (BID). 2020. "La educación superior en tiempos de COVID-19: Aportes de la segunda reunión del diálogo virtual con rectores de universidades líderes de América Latina". Acccedido: enero de 2021. https://bit.ly/3uA6qZG.

Casal Otero, Lorena, Carmen Fernández Morante y Beatriz Cebreiro López. 2018. "La competencia en TIC del profesorado no universitario". Revista Interuniversitaria de Investigación en Tecnología Educativa 5: 22-39. https://doi.org/10.6018/riite/2018/334851.

Castro, Alejandro. 2018. "La educación superior de Ecuador". Accedido enero de 2021: https://bit.ly/3vU3KXo.

Cedeño, Eva, y José Murillo. 2019. "Entornos virtuales de aprendizaje y su rol innovador en el proceso de enseñanza". Rehuso: Revista de Ciencias Humanísticas y Sociales" 4 (1): 119-127. https://bit.ly/3tvZ5s.

Cooperación Económica para América Latina (CEPAL). 2020. "La educación en tiempos de la pandemia de COVID-19". Accedido enero de 2021. https://bit.ly/3olinVK. 
EC Consejo de Educación Superior (CES). 2019. Reglamento de Régimen Académico. RPCSO-08-No.111-2019 Accedido febrero de 2021. https://bit.ly/3nYklX0.

Food and Agriculture Organization of the United Nations (FAO). 2014. "Metodologías de una guía para el diseño". Accedido enero de 2021. https://bit.ly/3er8ldv.

Fundación Universitaria Iberoamericana. 2006. Gestión del conocimiento y aprendizaje organizacional. Madrid: Siglo XXI.

García Muñoz, Aparicio, Cecilia Camacho Gómez y María del Carmen Ancona Alcocer. 2012. "El uso de las tecnologías digitales como un proceso educativo en la sociedad del conocimiento".Etic@net 1 (12): 83-95.https://bit.ly/33ohvBk.

Gómez, Martha Esthela, Leticia Contreras Orozco y Delia Gutiérrez Linares. 2016. "El impacto de las tecnologías de la información y la comunicación en estudiantes de ciencias sociales: un estudio comparativo de dos universidades públicas". Innovación Educativa 16 (71): 61-80. https://bit.ly/3xWtB2q.

Pérez, Julián, y María Merino. 2017. "Definición de aula virtual - qué es, significado y concepto". Accedido enero de 2021: https://definicion.de/aula-virtual/.

Sempértegui Ontaneda, Fernando. 2020. Entrevistado por Diego Oquendo. Radio Visión. "Entrevista a rector de la Universidad Central del Ecuador". En YouTube. Accedido febrero de $2021 \mathrm{https}: / /$ www.youtube.com/watch?v=4BsLsKfPScs.

Suasnabas, Lenín. 2020. "Calidad de la educación en Ecuador. ¿Mito o realidad? Dominio de las Ciencias 6 (2): 133-157. https://bit.ly/3vQg1fh.

UNESCO. 2008. Declaración mundial sobre educación para todos. Satisfacción de las necesidades básicas de aprendizaje. Accedido febrero de 2021. https://bit.ly/2YrBeRl.

---. 2013. Enfoques estratégicos sobre la TIC en educación en América Latina y el Caribe. Santiago: OREALC / UNESCO. https://bit.ly/33uBDBE.

Universidad Central de Ecuador (UCE) y Dirección de Desarrollo Académico. 2020. "El ciclo del aprendizaje en ambientes virtuales". Accedido febrero de 2020. https://bit.ly/2SxukqF.

Velandia, Lucy, Luis Gómez, Javier Piragauta y Celio Aros, 2018. Las TIC en la educación. El papel de las TIC en la transformación de la sociedad. Bogotá: Los Libertadores. https:// doi.org/10.2307/j.ctv11wjdp.4. 